\title{
High-flow nasal cannula oxygen therapy decreases postextubation neuroventilatory drive and work of breathing in patients with chronic obstructive pulmonary disease
}

Rosa Di mussi ${ }^{1}$, Savino Spadaro ${ }^{2}$, Tania Stripoli ${ }^{1}$, Carlo Alberto Volta ${ }^{2}$, Paolo Trerotoli ${ }^{3}$, Paola Pierucci ${ }^{4}$, Francesco Staffieri ${ }^{5}$, Francesco Bruno ${ }^{1}$, Luigi Camporota ${ }^{6}$ and Salvatore Grasso ${ }^{{ }^{*}}$ (i)

\begin{abstract}
Background: The physiological effects of high-flow nasal cannula $\mathrm{O}_{2}$ therapy (HFNC) have been evaluated mainly in patients with hypoxemic respiratory failure. In this study, we compared the effects of HFNC and conventional low-flow $\mathrm{O}_{2}$ therapy on the neuroventilatory drive and work of breathing postextubation in patients with a background of chronic obstructive pulmonary disease (COPD) who had received mechanical ventilation for hypercapnic respiratory failure.

Methods: This was a single center, unblinded, cross-over study on 14 postextubation COPD patients who were recovering from an episode of acute hypercapnic respiratory failure of various etiologies. After extubation, each patient received two 1-h periods of HFNC (HFNC1 and HFNC2) alternated with $1 \mathrm{~h}$ of conventional low-flow $\mathrm{O}_{2}$ therapy via a face mask. The inspiratory fraction of oxygen was titrated to achieve an arterial $\mathrm{O}_{2}$ saturation target of 88-92\%. Gas exchange, breathing pattern, neuroventilatory drive (electrical diaphragmatic activity (EAdi)) and work of breathing (inspiratory trans-diaphragmatic pressure-time product per minute (PTP DI/min $)$ ) were recorded.

Results: EAdi peak increased from a mean $( \pm$ SD) of $15.4 \pm 6.4$ to $23.6 \pm 10.5 \mu \mathrm{V}$ switching from HFNC1 to conventional $\mathrm{O}_{2}$, and then returned to $15.2 \pm 6.4 \mu \mathrm{V}$ during HFNC2 (conventional $\mathrm{O}_{2}: p<0.05$ versus HFNC1 and HFNC2). Similarly, the PTP DI/min increased from $135 \pm 60$ to $211 \pm 70 \mathrm{cmH}_{2} \mathrm{O} / \mathrm{s} / \mathrm{min}$, and then decreased again during HFNC2 to $132 \pm 56$ (conventional $\mathrm{O}_{2}: p<0.05$ versus HFNC1 and HFNC2).
\end{abstract}

Conclusions: In patients with COPD, the application of HFNC postextubation significantly decreased the neuroventilatory drive and work of breathing compared with conventional $\mathrm{O}_{2}$ therapy.

Keywords: High-flow nasal cannula oxygen therapy, Chronic obstructive pulmonary disease, Weaning from mechanical ventilation, Neuroventilatory drive, Work of breathing

\section{Background}

High-flow nasal cannula oxygen therapy (HFNC) consists of a totally conditioned, warmed, and humidified air/oxygen blend through a wide-bore nasal cannula at a flow rate between 20 and $60 \mathrm{~L} / \mathrm{min}$ [1]. Compared with the 'conventional' oxygen therapy devices, which deliver

\footnotetext{
* Correspondence: salvatore.grasso@uniba.it

'Dipartimento dell'Emergenza e Trapianti d'Organo (DETO), Sezione di

Anestesiologia e Rianimazione, Ospedale Policlinico, Università degli Studi di Bari "Aldo Moro", Piazza Giulio Cesare 11, Bari, Italy

Full list of author information is available at the end of the article
}

gas at $5-20 \mathrm{~L} / \mathrm{min}$ (conventional $\mathrm{O}_{2}$ ), during HFNC the tracheal inspiratory oxygen fraction $\left(\mathrm{FiO}_{2}\right)$ is more predictable [2] and the mucociliary function is better preserved [3]. In addition, HFNC generates a positive airway pressure (between 2 and $8 \mathrm{cmH}_{2} \mathrm{O}$ at the pharyngeal level) which resembles positive end-expiratory pressure (PEEP) and is proportional to the administered gas flow rate and varies with the patient breathing pattern (i.e., breathing with the mouth open or closed) [4]. Furthermore, HFNC results in a significant, flow-dependent ' $\mathrm{CO}_{2}$ wash out effect' of the nasopharyngeal space which decreases the

(c) The Author(s). 2018 Open Access This article is distributed under the terms of the Creative Commons Attribution 4.0 International License (http://creativecommons.org/licenses/by/4.0/), which permits unrestricted use, distribution, and 
anatomical dead space ventilation and therefore the $\mathrm{CO}_{2}$ rebreathing [5]. It seems likely that the overall impact of HFNC on the respiratory function results from the synergistic interaction of the mechanisms described above as well as other, more subtle, and as yet incompletely understood mechanisms [6].

Since its introduction, HFNC has been applied to treat patients with hypoxemic respiratory failure [2, 7-9] and to prevent reintubation in patients at risk of extubation failure $[10-12]$. In these patients, compared with conventional $\mathrm{O}_{2}$ therapy, HFNC improves oxygenation and decreases the work of breathing (WOB) $[10,13]$. Studies in patients with stable chronic obstructive pulmonary disease (COPD) in a home-care setting suggest favorable effects on the WOB and gas exchange [14-16]. However, far less known are the physiological effects of HFNC on neuroventilatory drive and WOB in patients with COPD in the critical care setting.

The electric activity of the diaphragm (EAdi) is a 'processed' diaphragmatic electromyography signal recorded through an array of electrode pairs mounted on the wall of a nasogastric feeding tube [17]. The EAdi is proportional to the intensity of the electrical stimuli directed to the diaphragm, i.e., the neuroventilatory drive [18-20]. Recently, Bellani and coworkers demonstrated that EAdi can be used to estimate the instantaneous WOB [21].

In this physiological study, we administered HFNC and conventional $\mathrm{O}_{2}$ therapy via a face mask postextubation in patients with a background of COPD who had received mechanical ventilation for hypercapnic respiratory failure from various etiologies. The hypothesis of this study was that, in these patients, HFNC decreases the neuroventilatory drive and WOB compared with conventional $\mathrm{O}_{2}$ therapy.

\section{Methods}

\section{Patient selection}

We enrolled patients with a background of moderate-severe COPD who were admitted to the intensive care unit (ICU) at the University Hospital of Bari (Italy) between December 2015 and December 2016 and required mechanical ventilation for acute hypercapnic ventilatory failure of various etiologies. The diagnosis of COPD was made by three experts, including one pulmonologist (PP) and two intensivists (SG and TS), and was graded in accordance with the Global Initiative for Chronic Obstructive Lung Disease (GOLD) criteria (http:// goldcopd.org/gold-2017-global-strategy-diagnosis-man agement-prevention-copd/). For each patient, the three assessors reviewed the clinical history, medical records, smoking history, frequency of exacerbation, spirometry data, radiological findings, physical examination, and measurement of static intrinsic PEEP on admission to ICU. The GOLD spirometry criterion for the diagnosis of COPD was a postbronchodilator forced expiratory volume in $1 \mathrm{~s} /$ forced vital capacity $\left(\mathrm{FEV}_{1} / \mathrm{FVC}\right)<0.7$. Based on the $\mathrm{FEV}_{1}$ impairment, the severity of COPD was defined as follows: GOLD stage 1 (mild), $\mathrm{FEV}_{1} \leq 80 \%$ predicted; GOLD stage 2 (moderate), $50 \% \leq \mathrm{FEV}_{1}<80 \%$ predicted; GOLD stage 3 (severe) $30 \% \leq \mathrm{FEV}_{1}<50 \%$ predicted; GOLD stage 4 (very severe), $\mathrm{FEV}_{1}<30 \%$ predicted. The local ethics committee approved the study protocol and informed consent requirements were met according to local regulations (Azienda Ospedaliero-Universitaria Policlinico di Bari Ethic Committee, protocol number: 885/C.E., May 2014).

Patients ready for extubation, as assessed by the treating clinician, were eligible for the study. According to our clinical protocol, the criteria defining readiness for extubation were: a) resolution or improvement of the condition leading to acute respiratory failure; b) set PEEP lower than $6 \mathrm{cmH}_{2} \mathrm{O}$ and $\mathrm{FiO}_{2}$ lower than 0.6 with a $\mathrm{PaO}_{2} / \mathrm{FiO}_{2}$ ratio greater than $150 \mathrm{mmHg}$; c) arterial $\mathrm{pH}>7.35$; c) Richmond Agitation Sedation Scale (RASS) between 0 and -1 [22], with no sedation or with a continuous infusion of dexmedetomidine $(0.1-1.4 \mu \mathrm{g} / \mathrm{kg} / \mathrm{h})$; and d) ability to trigger the ventilator, i.e., to decrease pressure airway opening $\left(\mathrm{P}_{\mathrm{AO}}\right)>3 \mathrm{cmH}_{2} \mathrm{O}$ during a brief (5-10 s) end-expiratory occlusion test. Other criteria included normothermia and hemodynamic stability unsupported by vasopressors or inotropes, but we allowed low-dose dobutamine $(<5 \mu \mathrm{g} / \mathrm{kg} / \mathrm{min})$ or low-dose dopamine $(<3 \mu \mathrm{g} / \mathrm{kg} / \mathrm{min})$. All patients underwent a 30-min spontaneous breathing trial (SBT) consisting of pressure support ventilation at $5 \mathrm{cmH}_{2} \mathrm{O}$ with a PEEP of 5 $\mathrm{cmH}_{2} \mathrm{O}$. Patients were eligible to be included in the study after a successful SBT.

Exclusion criteria were: age $<18$ years; $<48 \mathrm{~h}$ of invasive mechanical ventilation; presence of a tracheostomy; contraindications to the insertion of the EAdi catheter (e.g., recent upper gastrointestinal surgery, esophageal varices, esophageal trauma); and concomitant neurological or neuromuscular pathologies and/or known phrenic nerve dysfunction. We also excluded patients showing paradoxical abdominal movements or the use of accessory inspiratory muscles. The reason for the latter exclusion criterion is because the correlation between work of breathing and EAdi is valid only if the diaphragm contributes to approximately $75 \%$ of the overall WOB [23], and the calculation of WOB from EAdi may be inaccurate if the work carried out by the accessory inspiratory muscles is more than that of the diaphragm.

Before extubation, all patients were ventilated with a Servo-i ventilator (Maquet, Getinge group Critical Care, Solna, Sweden) equipped with the EAdi software (Maquet, Getinge group Critical Care, Solna, Sweden). At the beginning of the study, the standard feeding nasogastric tube was 
replaced with a 16-Fr, 125-cm EAdi catheter (Maquet, Getinge group Critical Care, Solna, Sweden) unless an EAdi catheter was already in place. The EAdi catheter was first positioned based on the corrected nose-earlobe-xyphoid distance formula, in accordance with the manufacturer's instructions [24]. Its position was subsequently adjusted using the ventilator EAdi catheter position tool (Servo-i ventilator NAVA software) [24].

\section{Measurements}

Patients were studied in the semirecumbent position. The EAdi signal was collected from the RS232 ventilator port at a sampling rate of $100 \mathrm{~Hz}$ (NAVA tracker software, Maquet Getinge group Critical Care, Solna, Sweden) and stored in a personal computer. The NAVA tracker files were subsequently converted and analyzed using the ICULab software package (Kleistek Engineering, Bari, Italy).

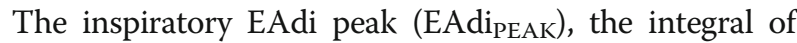
the inspiratory EAdi deflection over time (EAdi $\mathrm{PTP}_{\text {) }}$ ), the slope of the EAdi from the beginning of inspiration to the peak (EAdi SLOPE$_{\text {SOP }}$, the respiratory rate (RR), and the neural inspiratory time $\left(\mathrm{Ti}_{\mathrm{NEUR}}\right)$ were measured from the EAdi waveform [25].

Given that all patients were breathing spontaneously, tidal volume (VT) was not measured to avoid any modification in breathing pattern caused by the measurement apparatus.

The pressure generated by the diaphragm (i.e., the trans-diaphragmatic pressure, $\mathrm{P}_{\mathrm{DI}}$ ) throughout the inspiratory phase was calculated from the EAdi signal as described by Bellani and coworkers [21]. Briefly, we calculated first the diaphragmatic neuromuscular efficiency (NME), i.e., the ratio between the negative peak in airway opening pressure $\left(\mathrm{P}_{\mathrm{AO}}\right)$ during a spontaneous inspiratory effort (recorded during a brief end-expiratory occlusion lasting 5-10 s) and the corresponding peak in the EAdi curve $[19,26,27]$. Since the fall in $\mathrm{P}_{\mathrm{AO}}$ during a spontaneous inspiratory effort against the occluded airways is, by definition, equal to the corresponding fall in esophageal pressure $\left(\mathrm{P}_{\mathrm{ES}}\right)[28,29]$, the $\mathrm{NME}$ is an index of diaphragmatic neuromechanical coupling, and accordingly can be used as a factor to convert the EAdi into $\mathrm{P}_{\mathrm{DI}}$
$\left(\mathrm{P}_{\mathrm{DI}}=\mathrm{EAdi} \times \mathrm{NME}\right)[21]$. The inspiratory $\mathrm{P}_{\mathrm{DI}}$ pressure-time product per breath $\left(\mathrm{PTP}_{\mathrm{DI} / \mathrm{b}}\right)$ was calculated as the area under the $\mathrm{P}_{\mathrm{DI}}$ signal. The inspiratory $\mathrm{P}_{\mathrm{DI}}$ pressure-time product per minute $\left(\mathrm{PTP}_{\mathrm{DI} / \mathrm{min}}\right)$ was calculated as:

$$
\mathrm{PTP}_{\mathrm{DI} / \min }=\mathrm{PTP}_{\mathrm{DI} / \mathrm{b}} \times \mathrm{RR} .
$$

\section{Study protocol}

At the beginning of the study, 5-10 min before extubation, the NME was calculated after a brief (5-10 s) end-expiratory occlusion. Immediately after extubation, patients underwent a cross-over protocol with an $\mathrm{ON}-$ OFF-ON design, alternating HFNC with conventional $\mathrm{O}_{2}$ delivered through a face mask (i.e., HFNC1 - conventional $\mathrm{O}_{2}$ therapy - HFNC2), with each phase lasting for $1 \mathrm{~h}$ (Fig. 1).

The HFNC was administered through the AIRVO 2 system (Fisher \& Paykel Healthcare, Auckland, New Zealand) and specific medium/large nasal prongs to fit the size of the nostrils (Fisher \& Paykel Healthcare, Auckland, New Zealand). The system allows for the administration of humidified and warmed gas flow (10$60 \mathrm{~L} / \mathrm{min}$ in the adult configuration). The gas flow was titrated upwards at 5-10 L/min steps starting from $20 \mathrm{~L} / \mathrm{min}$, up to the highest flow compatible with patient comfort (maximum allowed flow $60 \mathrm{~L} / \mathrm{min}$ ) [30]. The $\mathrm{FiO}_{2}$ was titrated to achieve an hemoglobin oxygen saturation $\left(\mathrm{SaO}_{2}\right)$ target of $88-92 \%$. The temperature of the heated humidifier was set at $37{ }^{\circ} \mathrm{C}$.

In keeping with previous studies [13], the conventional $\mathrm{O}_{2}$ therapy was administered through a standard nonocclusive oxygen facial mask connected to a $\mathrm{O}_{2} /$ air mixer $(0-20 \mathrm{~L} / \mathrm{min})$. The mask gas flow was set to $10 \mathrm{~L} / \mathrm{min}$ in all the patients. The $\mathrm{FiO}_{2}$ in the mask flow was titrated to achieve the $88-92 \% \mathrm{SaO}_{2}$ target.

At the end of each study period, arterial blood gas analysis was performed. EAdi and $\mathrm{P}_{\mathrm{DI}}$ parameters were calculated from the digital recordings of EAdi curve on the last $30 \mathrm{~min}$ of each step.

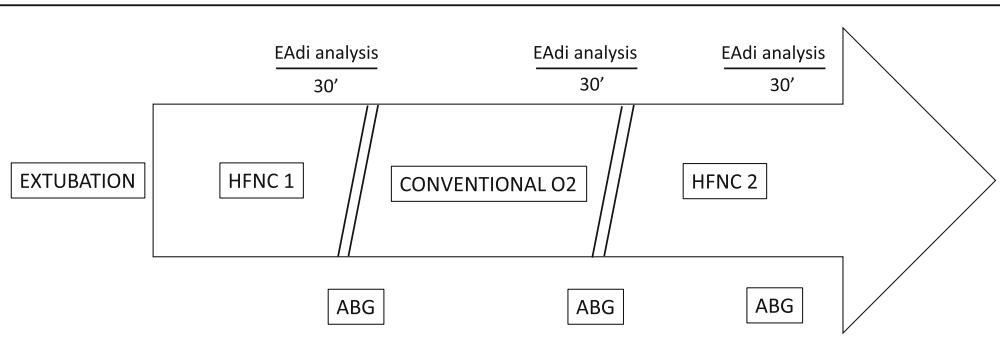

Fig. 1 Study protocol timeline. ABG arterial blood gas, Conventional $\mathrm{O}_{2}$ period of conventional low flow oxygen therapy through a non-occlusive face mask, EAdi diaphragm electrical activity, HFNC1 first period of high flow nasal cannula oxygen therapy, HFNC2 second period of high flow nasal cannula oxygen therapy 
Extubation success was defined as the ability of the patient to breathe spontaneously without signs of respiratory distress and without the requirement of rescue noninvasive ventilation (NIV) for $48 \mathrm{~h}$ postextubation. Signs of respiratory distress were defined as: a) paradoxical abdominal movement, use of accessory respiratory muscles, or evidence of respiratory muscle fatigue; b) cardiovascular instability (systolic blood pressure (SBP) > 160 or $<90 \mathrm{mmHg}$ or a $20 \%$ change from the pre-SBT values; heart rate $(\mathrm{HR})>120$ or $<60$ beats $/ \mathrm{min}$ or $20 \%$ change from the pre-SBT values; c) arterial desaturation with $\mathrm{SaO}_{2}<88 \%$ ), hypercapnia, and respiratory acidosis with $\mathrm{pH}<7.35$; and $\mathrm{d}$ ) retention of secretions.

\section{Statistical analysis}

The power analysis indicated a sample size of 14 patients with a power of 0.8 , a significance level of 0.05 and an expected effect size of 0.25 . The effect size refers to the magnitude of variability in an outcome explained by the intervention divided by the total variability of the same outcome measure. We have hypothesized that the variability explained by the study condition in EAdi PEAK $_{\text {had }}$ to be at least $5 \%$ of total variability that corresponds approximatively to a medium effect size of 0.25 . A sphericity correction of 0.8 and a correlation of 0.8 were assumed from pilot measurements. The sample size was determined using the software GPower version 3.1.9.2.

Continuous quantitative variables were summarized as mean \pm standard deviation (SD) if normally distributed or as median and interquartile range if non-normally distributed. Comparisons were performed with analysis of variance (ANOVA) for repeated measures or Friedman's test as appropriate. A $p$ value $<0.05$ was considered statistically significant, except in the multiple comparison procedure, when the $p$ value was adjusted. The analyses were carried out with SAS software v.9.4 for Windows PC.

\section{Results}

The CONSORT diagram of our study (Fig. 2) shows that 20 out of the 57 COPD patients admitted to our unit during the study period were eligible for the study and 16 were enrolled. Two patients were excluded from the final analysis because of technical difficulties in recording the EAdi signal. Therefore, the final number of patients was 14. Patient demographics and clinical characteristics are shown in Table 1.

Five patients $(31.5 \%)$ failed the initial extubation attempt and were reintubated. Patients who required reintubation were similar to the ones who were successfully

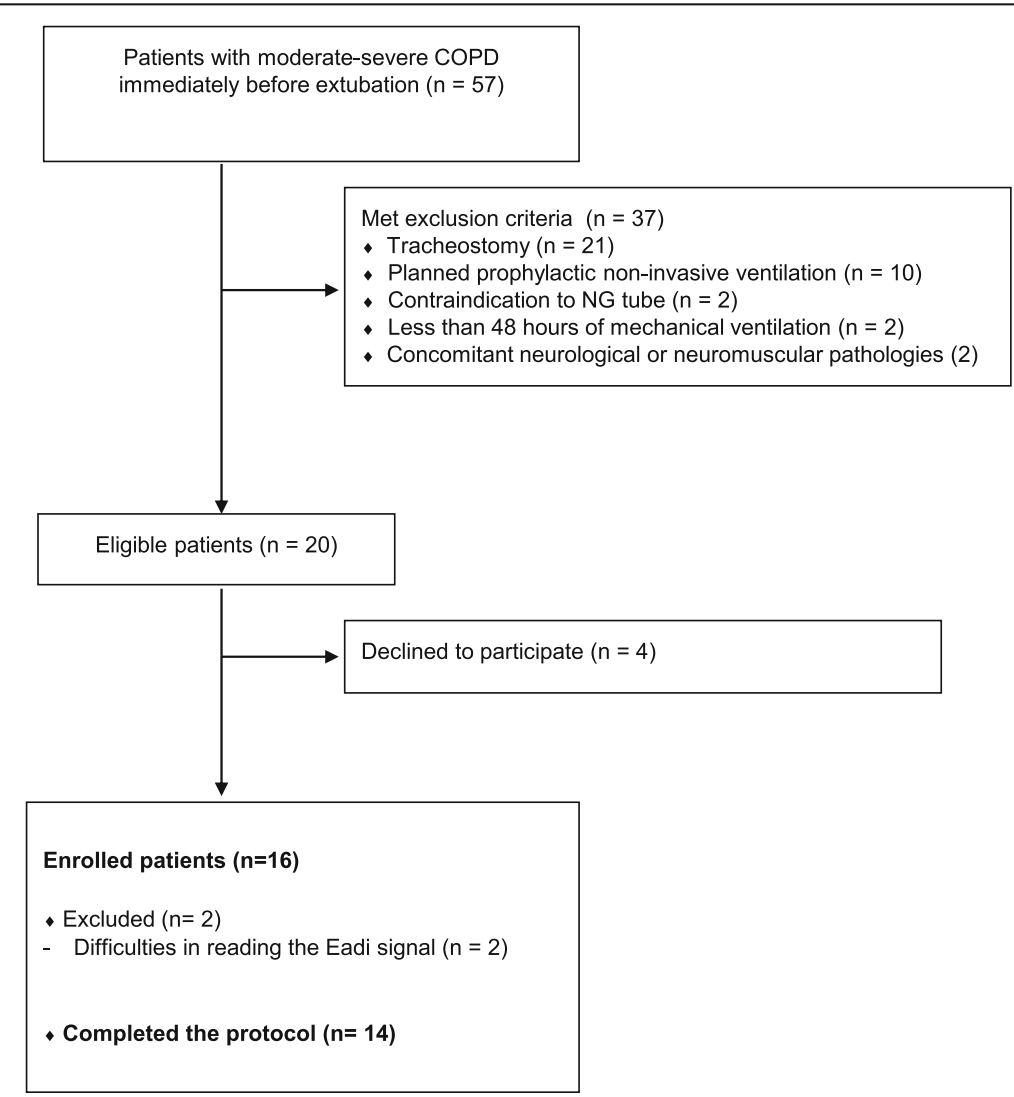

Fig. 2 Flow diagram of patient enrollment. COPD chronic obstructive pulmonary disease, EAdi diaphragm electrical activity, NG nasogastric 


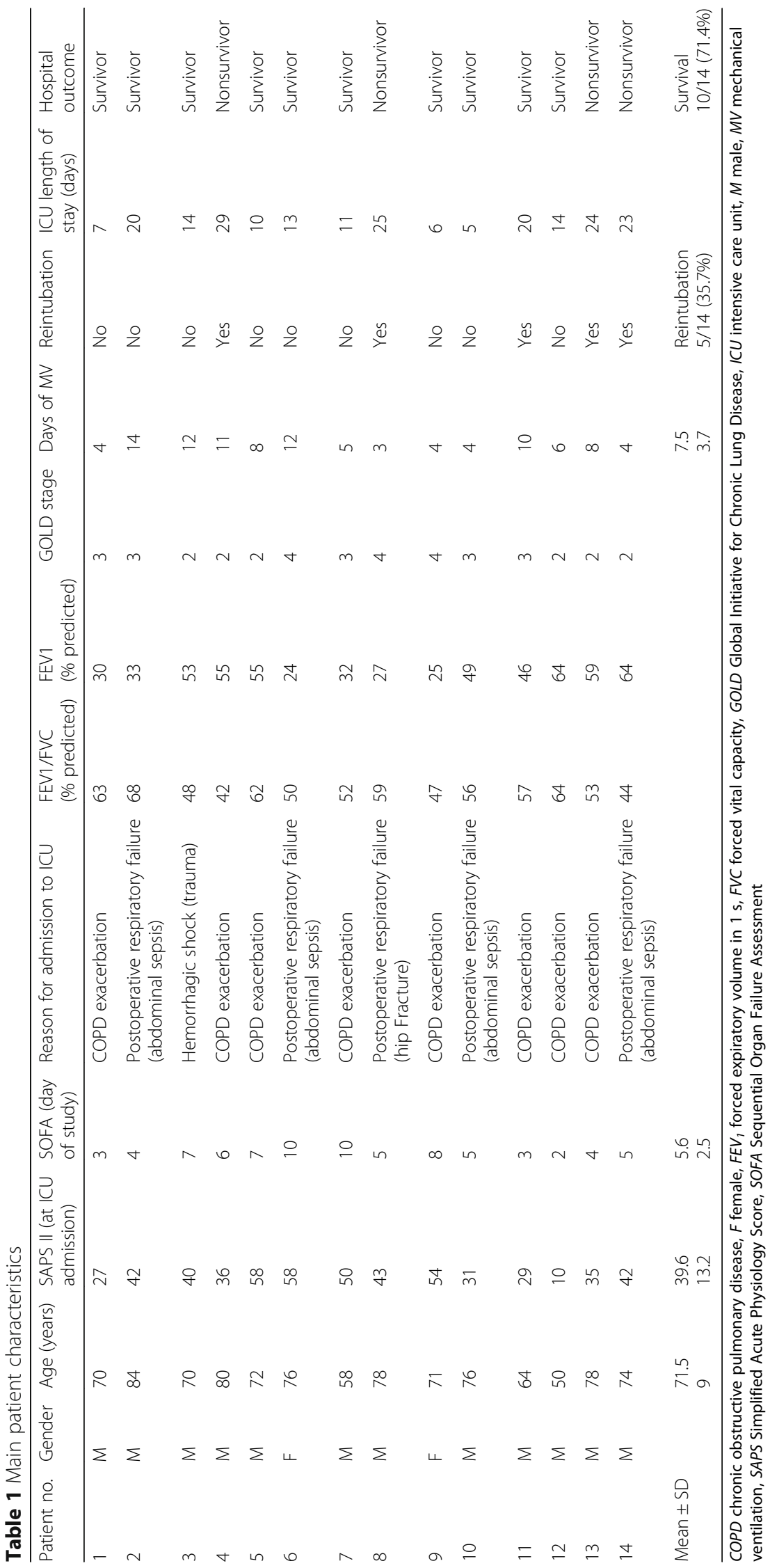


extubated in terms of age, reason for ICU admission (COPD exacerbation vs other causes), days of mechanical ventilation, COPD severity (based on $\mathrm{FEV}_{1}, \mathrm{FEV}_{1}$ / FVC ratio, GOLD stage, Simplified Acute Physiology Score (SAPS) II on admission, and Sequential Organ Failure Assessment (SOFA) score; Additional file 1).

Four patients $(25 \%)$ died after a mean $( \pm S D)$ ICU length of stay of $25.2 \pm 2.6$ days. The cause of death for two patients was septic shock and multiple organ failure, while the other two died of right cardiac failure and cardiogenic shock.

\section{Breathing pattern and gas exchange}

Table 2 shows the breathing pattern and gas exchange recorded for each of the three experimental conditions. To achieve the oxygenation target $\left(\mathrm{SaO}_{2}\right.$ between 88 and $\left.92 \%\right)$, the applied $\mathrm{FiO}_{2}$ during HFNC1 and HFNC2 periods was $0.46 \pm 0.1$ and $0.46 \pm 0.12$, respectively ( $p=$ not significant), whereas during the conventional (mask) $\mathrm{O}_{2}$ period it was $0.80 \pm 0.19$. However, a comparison between the $\mathrm{FiO}_{2}$ during HFNC and conventional $\mathrm{O}_{2}$ is not meaningful given the difference in the delivered gas flow rates. Indeed, the $\mathrm{FiO}_{2}$ delivered during conventional $\mathrm{O}_{2}$ therapy is almost certainly overestimated since the patient's inspiratory flow is higher than the mask flow $(10 \mathrm{~L} / \mathrm{min})$ and, therefore, the difference between patient inspiratory flow and the mask flow is provided by room air. This also makes any comparison between the $\mathrm{PaO}_{2} / \mathrm{FiO}_{2}$ in the different experimental conditions inappropriate. Respiratory rate, $\mathrm{Ti}_{\mathrm{NEUR}}$, arterial $\mathrm{PCO}_{2}$, and $\mathrm{pH}$ remained similar throughout the study (Table 2).

Table 2 Breathing pattern and gas exchange in different experimental conditions

\begin{tabular}{llll}
\hline & HFNC1 & Conventional $\mathrm{O}_{2}$ & HFNC2 \\
\hline RR (breaths/min) & $20.5 \pm 2.9$ & $21.4 \pm 4$ & $20.0 \pm 1.9$ \\
$\mathrm{Ti}_{\text {NEUR }}(\mathrm{s})$ & $0.92 \pm 0.21$ & $0.95 \pm 0.22$ & $0.92 \pm 0.17$ \\
$\mathrm{pH}$ & $7.45 \pm 0.07$ & $7.44 \pm 0.08$ & $7.46 \pm 0.08$ \\
$\mathrm{PaCO}_{2}(\mathrm{mmHg})$ & $49.9 \pm 11.9$ & $51.8 \pm 12.7$ & $50.1 \pm 12.6$ \\
$\mathrm{HCO}_{3}{ }^{-}(\mathrm{mEq} / \mathrm{L})$ & $30.9 \pm 7.6$ & $31.3 \pm 7.8$ & $31.4 \pm 8.4$ \\
$\mathrm{PaO}_{2}(\mathrm{mmHg})$ & $75.1 \pm 6.9$ & $72.9 \pm 8.6$ & $81.2 \pm 8$ \\
Applied FiO $_{2}{ }^{\mathrm{a}}$ & $0.46 \pm 10$ & $0.80 \pm 0.19^{\mathrm{b}, \mathrm{c}}$ & $0.46 \pm 0.12$ \\
\hline
\end{tabular}

Data are expressed as mean \pm standard deviation

Conventional $\mathrm{O}_{2}$ conventional low flow oxygen therapy through a nonocclusive face mask, $\mathrm{FiO}_{2}$ inspiratory oxygen fraction, $\mathrm{HFNC}$ high-flow nasal cannula oxygen therapy, $\mathrm{PaCO}_{2}$ arterial partial carbon dioxide pressure, $\mathrm{PaO}_{2}$ arterial partial oxygen pressure, $R R$ respiratory rate, $T i_{N E U R}$ neural inspiratory time

${ }^{a}$ The $\mathrm{FiO}_{2}$ delivered during conventional $\mathrm{O}_{2}$ therapy is overestimated since the patient's inspiratory flow was higher than the mask flow $(10 \mathrm{~L} / \mathrm{min})$ and, therefore, the difference between patient inspiratory flow and mask flow was taken by room air; this makes inappropriate any comparison between the $\mathrm{PaO}_{2} / \mathrm{FiO}_{2}$ ratio in the different experimental conditions

${ }^{b}$ Different from HFNC1, ANOVA, with Bonferroni correction

${ }^{c}$ Different from HFNC2, ANOVA, with Bonferroni correction

\section{Neuroventilatory drive and work of breathing}

Figure 3 shows EAdi traces obtained at the end of each study period in three representative patients. In all patients, the neuroventilatory drive, expressed by the EAdi waveforms, clearly increased between HFNC1 and conventional $\mathrm{O}_{2}$ therapy and decreased again when the HFNC was reinstituted (HFNC2). Table 3 shows that neuroventilatory drive (EAdi $\left.\mathrm{EEAK}_{\mathrm{PEK}}\right)$ and work of breathing $\left(\mathrm{PTP}_{\mathrm{DI} / \mathrm{b}}\right.$ and $\mathrm{PTP}_{\mathrm{DI} / \mathrm{min}}$ ) increased significantly while on conventional $\mathrm{O}_{2}$ therapy, and decreased again when HFNC was reinstituted. Figure 4 shows the individual changes in these parameters during the three study periods.

We were not able to find any significant differences between patients that were reintubated and patients successfully extubated in terms of EAdi parameters or work of breathing during each experimental condition (Additional file 2). For the same parameters, there were no significant differences between patients admitted for hypercapnic respiratory failure due to an exacerbation of COPD and patients with a background of COPD but whose hypercapnic respiratory failure was due to other precipitating causes (Additional file 3).

\section{Discussion}

This study shows that postextubation HFNC significantly decreases the neuroventilatory drive and work of breathing in patients with COPD who had received mechanical ventilation for hypercapnic respiratory failure due to various etiologies.

The EAdi reflects the rate of discharge of the phrenic nerve and therefore it is a measure of the neuroventilatory drive $[17-20,31,32]$. Thus, our data clearly show that HFNC decreases the neuroventilatory drive (EAdiPEAK and EAdi SLOPE$_{\text {) }}$ compared with conventional $\mathrm{O}_{2}$ therapy. Neuroventilatory drive and work of breathing are key factors for the weaning process and an excessive respiratory drive predicts weaning failure [26, 32]. In fact, a high ventilatory drive may be associated either with excessive mechanical load posed on the inspiratory muscles, diaphragm weakness, or inappropriately high activation of the respiratory centers due to pain, fever, anxiety, and acidosis [32]. In a mixed population of critically ill patients, Liu and coworkers found that an EAdiPEAK lower than 15-20 $\mu \mathrm{V}$ during a spontaneous breathing trial (T-tube) was associated with weaning success [26]. Similar results were recently obtained in two other studies by Dres et al. [33] and Barwing et al. [34]. In our study, we found that the EAdiP $P_{\text {EAK }}$ was below this threshold in most of the patients during both HFNC periods (Fig. 4), while it was on average 1.5-times higher than this threshold during conventional $\mathrm{O}_{2}$. Accordingly, considering that COPD patients are intrinsically at risk of weaning failure [35], our results are potentially clinically relevant. 


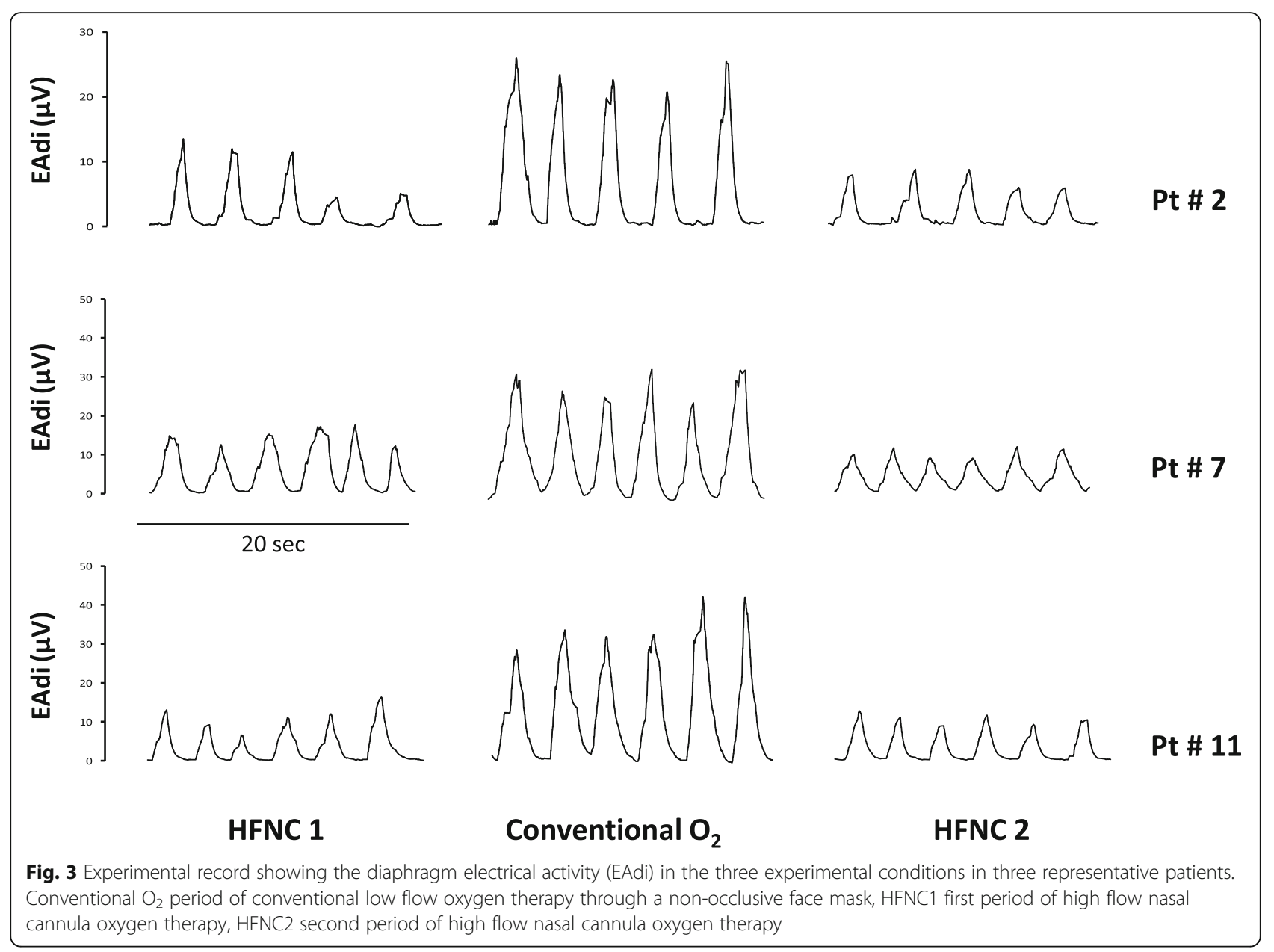

Although the work of breathing is proportional to the neuroventilatory drive, its absolute value depends on the ability of the respiratory muscles to convert the electrical stimuli into mechanical contraction (electromechanical coupling) [18, 32]. We measured the work of breathing in terms of $\mathrm{PTP}_{\mathrm{DI}}$ per breath and per minute, a

Table 3 Neuroventilatory drive and work of breathing parameters

\begin{tabular}{llll}
\hline & HFNC1 & Conventional $\mathrm{O}_{2}$ & HFNC2 \\
\hline $\operatorname{EAdi}_{\text {PEAK }}(\mu \mathrm{V})$ & $15.4 \pm 6.4$ & $23.6 \pm 10.5^{\mathrm{a}, \mathrm{b}}$ & $15.2 \pm 6.4$ \\
EAdi $_{\text {PTP }}(\mu \mathrm{V} / \mathrm{s})$ & $13.7 \pm 6.5$ & $21.1 \pm 11.8^{\mathrm{a}, \mathrm{b}}$ & $12.1 \pm 5.2$ \\
EAdi $_{\text {SLOPE }}$ & $18.6 \pm 6.5$ & $24 \pm 14.7^{\mathrm{a}, \mathrm{b}}$ & $17.6 \pm 10.2$ \\
$\operatorname{PTP}_{\text {DI/b }}\left(\mathrm{cmH}_{2} \mathrm{O} / \mathrm{s}\right)$ & $6.7 \pm 2.7$ & $9.9 \pm 3.1^{\mathrm{a}, \mathrm{b}}$ & $6.7 \pm 2.8$ \\
PTP $_{\text {DI/min }}\left(\mathrm{cmH}_{2} \mathrm{O} / \mathrm{s} / \mathrm{min}\right)$ & $135 \pm 60$ & $211 \pm 70^{\mathrm{a}, \mathrm{b}}$ & $132 \pm 56$ \\
\hline
\end{tabular}

\section{Data are expressed as mean \pm standard deviation}

Conventional $\mathrm{O}_{2}$ conventional low flow oxygen therapy through a nonocclusive face mask, EAdi ${ }_{P E A K}$ diaphragm electrical activity peak, EAdi $i_{P T P}$ EAdi deflection inspiratory area, EAdi ${ }_{\text {SLOPE }}$ EAdi slope from the beginning of inspiration to EAdi PEAK, $_{\text {, }}$ HFNC high-flow nasal cannula oxygen therapy, PTP inspiratory trans-diaphragmatic pressure-time product per breath, $P T P_{D / / m i n}$ inspiratory trans-diaphragmatic pressure-time product per minute

${ }^{\text {a }}$ Different from HFNC1, ANOVA, with Bonferroni correction

${ }^{b}$ Different from HFNC2, ANOVA, with Bonferroni correction well-known index of respiratory muscle oxygen consumption (Table 3 and Fig. 4). According to physiological studies in mixed populations of critically ill patients, an 'acceptable' $\mathrm{PTP}_{\mathrm{DI} / \mathrm{min}}$ is between 50 and $150 \mathrm{cmH}_{2} \mathrm{O} / \mathrm{s} / \mathrm{min}[36,37]$. The $\mathrm{PTP}_{\mathrm{DI} / \mathrm{min}}$ was in this range in $64.3 \%$ of our patients (i.e., 9/14) both during HFNC1 and HFNC2 periods, whereas the $\mathrm{PTP}_{\mathrm{DI} / \mathrm{min}}$ was above this acceptable range in $78.6 \%$ of patients during the conventional $\mathrm{O}_{2}$ period (i.e., 11/14) (Fig. 3).

According to the 2017 European Respiratory SocietyAmerican Thoracic Society (ERS/ATS) guidelines [38], COPD patients benefit from noninvasive ventilation to prevent reintubation. Therefore, it would have been of interest to compare the physiological effects of HFNC and NIV in our patients. However, at the time of the study, postextubation preventative NIV was not applied on a routine basis in our institution. Interestingly, a recent study by Hernandez et al. showed that HFNC is noninferior to NIV in preventing acute postextubation respiratory failure in patients at "high risk" of postextubation respiratory failure, including patients older than 65 years or those with heart failure, moderate to severe COPD, an Acute Physiology and Chronic Health Evaluation (APACHE) II 


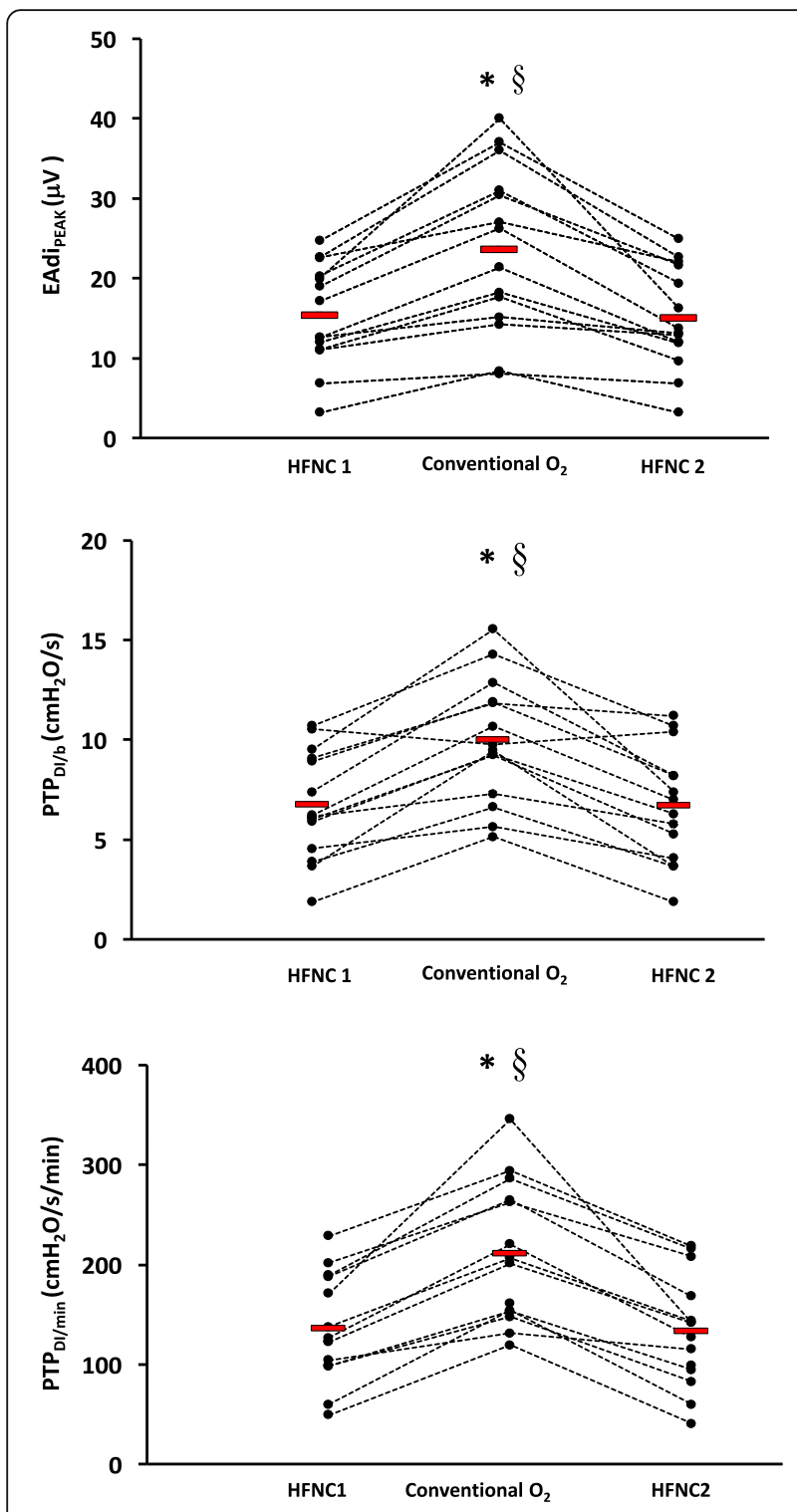

Fig. 4 Trend of the neuroventilatory drive, as expressed by the diaphragm electrical activity peak EAdi breathing, as expressed by the inspiratory $P_{D I}$ pressure-time product per breath (PTP difference compared to the HFNC1 period (ANOVA with Bonferroni correction); \$Significant difference compared to the HFNC2 period (ANOVA with Bonferroni correction). Conventional $\mathrm{O}_{2}$ period of conventional low flow oxygen therapy through a non-occlusive face mask, HFNC1 first period of high flow nasal cannula oxygen therapy, HFNC2 second period of high flow nasal cannula oxygen therapy

score higher than 12 on extubation day, a body mass index of more than 30 , those with airway patency problems, and, finally, patients with difficult or prolonged weaning [11].

Further studies are needed to assess the beneficial mechanisms of HFNC in COPD patients. We speculate that two mechanisms are of particular relevance: a) the HFNC "PEEP" effect [14], that may have counterbalanced the flow-limited intrinsic positive end-expiratory pressure
(PEEPi), and b) the " $\mathrm{CO}_{2}$ wash-out" effect of the anatomical dead space [5] that may have decreased the diaphragmatic workload. The better preservation of the mucociliary function as compared with conventional $\mathrm{O}_{2}$ therapy may have been an adjunctive mechanism [3], but we believe that it was less important since the cross-over periods were relatively short.

In hypoxemic patients, Mauri et al. [39] and Maggiore et al. [10] found that HFNC significantly decreased RR compared with conventional $\mathrm{O}_{2}$ therapy. Mauri estimated the VT through electrical impedance tomography (EIT) and found that it remained stable. In contrast, in our COPD patients, the RR remained unchanged (Table 2), while we have no data on VT since patients were breathing spontaneously and we wanted to avoid any modification in breathing pattern caused by the measurement apparatus. However, the VT likely increased since animal studies show that VT is proportional to the electrical activity of the diaphragm during unassisted spontaneous breathing [40]. Based on this hypothesis, in our patients, the response to $\mathrm{HFNC}$ removal during the conventional $\mathrm{O}_{2}$ period would have been similar to the physiological response to a sudden increase in respiratory workload during to $\mathrm{CO}_{2}$ rebreathing, i.e., to maintain the $\mathrm{RR}$ as constant and to increase the VT $[41,42]$. The different impact of HFNC on RR between our study and those of Mauri and Maggiore could be explained by the different background of the respiratory failure of the studied patients (hypoxemic versus hypercapnic).

In our study, similar to previous studies [13], we used a standard, nonocclusive oxygen facial mask with a fixed gas flow of $10 \mathrm{~L} / \mathrm{min}$ in all the patients during the conventional $\mathrm{O}_{2}$ study step (see the Methods section). Hence, in our patients the peak inspiratory flow was very likely greater than the mask gas flow and therefore the true fraction of inhaled oxygen was lower than the one provided by the mask. The "Venturi Mask" is a high-flow oxygen delivery system that provides 35-45 L/min of a mixture of oxygen and air with a delivered $\mathrm{FiO}_{2}$ of $0.24-0.6$ by taking advantage of the Bernoulli principle [43]. By using a Venturi Mask instead of the standard mask, it is possible that we would have better matched the patient's inspiratory flow during the conventional $\mathrm{O}_{2}$ study period. One could also speculate that a higher mask flow could have other effects in terms of $\mathrm{CO}_{2}$ washout from the mask or from the airways, but we are not aware of studies comparing Venturi mask and HFNC.

We must acknowledge some study limitations. First, we studied a population of patients with COPD that was admitted to the ICU with hypercapnic ventilatory failure due to various etiologies (Table 1 ). Only 8/14 (57\%) of COPD patients were admitted because of a COPD exacerbation, while the other $6(43 \%)$ received mechanical ventilation for postoperative ventilatory failure. In this regard, our population could be deemed as heterogenous. However, 
we point out that: 1) our study was conducted in the postextubation phase when the primary reason for the acute respiratory failure had resolved or at least improved (see Methods); and 2) all our patients had moderate to very severe COPD according to the GOLD classification. Second, we were not able to measure several respiratory parameters during spontaneous breathing (VT, PEEPi, inspiratory flow) that could have provided us with a more complete interpretation of the treatment effect. However, our study was conducted in spontaneously breathing patients and we sought to avoid any modification in breathing pattern caused by the measurement apparatus. Third, we measured the work of breathing based on a method recently validated by Bellani and coworkers [21], but the correlation between work of breathing and EAdi may be misleading if the contraction of the accessory inspiratory muscles is dominant compared with the diaphragmatic contraction. Indeed, the estimation of work of breathing from EAdi assumes that the diaphragm contributes approximately $75 \%$ to the overall WOB (as occurs in normal conditions) [23]. However, we assessed all patients for signs of paradoxical abdominal motion and use of accessory inspiratory muscles throughout the study. In addition, the method described by Bellani et al. assumes a linear relationship between EAdi and $\mathrm{P}_{\mathrm{DI}}$ at different lung volumes based on a close correlation at different lung volumes between the $\mathrm{P}_{\mathrm{DI}}$ obtained from the esophageal pressure and the $\mathrm{P}_{\mathrm{DI}}$ obtained through the formula EAdi $\times$ NME [21]. However, Bellani et al. studied patients ventilated with different levels of pressure support ventilation (PSV) and neurally adjusted ventilatory assist (NAVA) while we studied spontaneously breathing patients. Of note, other authors showed a nonlinearity between diaphragmatic efficiency and lung volumes, but only for intense diaphragmatic contractions [19]. Fourth, we studied a small patient number that, while appropriate for a physiologically oriented study, weakens any speculation on the clinical outcomes (e.g., ICU and hospital length of stay and reintubation rate).

\section{Conclusions}

In conclusion, we found that HFNC, as compared with conventional $\mathrm{O}_{2}$ therapy, significantly decreases the neuroventilatory drive and the work of breathing in patients with COPD recovering from an episode of acute respiratory failure after a planned extubation.

\section{Additional files}

Additional file 1: Independent sample $t$ tests. Comparison between patients who required reintubation and patients who were successfully extubated in terms of age, reason for ICU admission (COPD exacerbation versus other causes), days of mechanical ventilation, COPD severity (based on $\mathrm{FEV}_{1}$, FEV, /FVC ratio, GOLD stage, SAPS II on admission, and SOFA score). (DOCX $26 \mathrm{~kb}$ )

Additional file 2: Independent sample $t$ tests. Comparison between patients who required reintubation and patients who were successfully extubated in terms of EAdi parameters or work of breathing during each experimental condition. (DOCX $42 \mathrm{~kb}$ )

Additional file 3: Independent sample $t$ tests. Comparison between patients admitted for hypercapnic respiratory failure due to an exacerbation of COPD and patients with a background of COPD but whose hypercapnic respiratory failure was due to other precipitating causes in terms of EAdi parameters or work of breathing during each experimental condition. (DOCX $60 \mathrm{~kb}$ )

\section{Abbreviations}

COPD: Chronic obstructive pulmonary disease; EAdi: Electric activity of the diaphragm; $\mathrm{FEV}_{1}$ : Forced expiratory volume in $1 \mathrm{~s} ; \mathrm{FiO}_{2}$ : Inspiratory oxygen fraction; FVC: Forced vital capacity; GOLD: Global Initiative for Chronic Lung Disease; HFNC: High-flow nasal cannula oxygen therapy; ICU: Intensive care unit; NIV: Noninvasive ventilation; NME: Neuromuscular efficiency; $\mathrm{P}_{\mathrm{AO}}$ : Pressure airway opening; PEEP: Positive end-expiratory pressure; PEEPI: Intrinsic positive end-expiratory pressure; PTP product per breath; $\mathrm{PTP}_{\mathrm{D} / \mathrm{min}}$ : Pressure-time product per minute; RR: Respiratory rate; $\mathrm{SaO}_{2}$ : Arterial hemoglobin oxygen saturation; SBP: Systolic blood pressure; SBT: Spontaneous breathing trial; Ti NEUR: Neural inspiratory time; VT: Tidal volume; WOB: Work of breathing

\section{Acknowledgements}

We would like to thank Dr. Joanna Brown for reviewing the final manuscript.

Funding

No specific funding was received for this study.

\section{Availability of data and materials}

The data that support the findings of this study are available from the corresponding author upon reasonable request.

\section{Authors' contributions}

SG, RDm, and SS contributed to the design of the conception and design of the study. RDm, SS, TS, and PP were responsible for patient screening and enrollment. PT performed the statistical analysis. SG, CAV, FB, LC, and FS analyzed the data and wrote the manuscript. All authors contributed to interpretation of the data. All authors read and approved the final manuscript.

\section{Ethics approval and consent to participate}

Ethical approval was obtained by the local ethics committee (Azienda Ospedaliero-Universitaria Policlinico di Bari Ethic Committee, protocol number: 885/C.E., May 2014). All patients or their relatives provided informed consent to participate.

\section{Consent for publication}

Not applicable.

\section{Competing interests}

SG has given talks in symposia organized by Getinge Critical Care (Solna Sweden), Estor Critical Care (Mirandola, Italy), and Fisher and Paykel (Auckland, New Zealand), and has received compensation for related travel and accommodation fees. He declares no other relationship or activities that could appear to have influence the submitted work. The remaining authors declare that they have no competing interests.

\section{Publisher's Note}

Springer Nature remains neutral with regard to jurisdictional claims in published maps and institutional affiliations.

\section{Author details}

'Dipartimento dell'Emergenza e Trapianti d'Organo (DETO), Sezione di Anestesiologia e Rianimazione, Ospedale Policlinico, Università degli Studi di Bari "Aldo Moro", Piazza Giulio Cesare 11, Bari, Italy. ${ }^{2}$ Dipartimento di 
Morfologia, Chirurgia e Medicina Sperimentale, Sezione di Anestesiologia e Terapia Intensiva Universitaria, Università degli studi di Ferrara, Ferrara, Italy. ${ }^{3}$ Dipartimento di Scienze Biomediche ed Oncologia Umana, Cattedra di Statistica Medica, Università degli Studi Aldo Moro, Bari, Italy. ${ }^{4}$ Dipartimento di Medicina Respiratoria e del Sonno, Università degli Studi di Bari "Aldo Moro", Bari, Italy. 'Dipartimento dell'Emergenza e Trapianti d'Organo (DETO), Sezione di Chirurgia Veterinaria, Università degli Studi di Bari "Aldo Moro", Bari, Italy. "Department of Adult Critical Care, Guy's and St Thomas' NHS Foundation Trust, King's Health Partners, and Division of Centre of Human Applied Physiological Sciences, King's College London, London, UK.

\section{Received: 6 April 2018 Accepted: 22 June 2018} Published online: 02 August 2018

\section{References}

1. Papazian L, Corley A, Hess D, Fraser JF, Frat JP, Guitton C, Jaber S, Maggiore SM, Nava S, Rello J, et al. Use of high-flow nasal cannula oxygenation in ICU adults: a narrative review. Intensive Care Med. 2016;42(9):1336-49.

2. Sztrymf B, Messika J, Bertrand F, Hurel D, Leon R, Dreyfuss D, Ricard JD Beneficial effects of humidified high flow nasal oxygen in critical care patients: a prospective pilot study. Intensive Care Med. 2011;37(11):1780-6.

3. Spoletini G, Alotaibi M, Blasi F, Hill NS. Heated humidified high-flow nasal oxygen in adults: mechanisms of action and clinical implications. Chest. 2015;148(1):253-61.

4. Parke RL, McGuinness SP. Pressures delivered by nasal high flow oxygen during all phases of the respiratory cycle. Respir Care. 2013;58(10):1621-4.

5. Moller W, Feng S, Domanski U, Franke KJ, Celik G, Bartenstein P, Becker S, Meyer G, Schmid O, Eickelberg O, et al. Nasal high flow reduces dead space. J Appl Physiol (1985). 2017;122(1):191-7.

6. Goligher EC, Slutsky AS. Not just oxygen? Mechanisms of benefit from highflow nasal cannula in hypoxemic respiratory failure. Am J Respir Crit Care Med. 2017;195(9):1128-31.

7. Messika J, Ben Ahmed K, Gaudry S, Miguel-Montanes R, Rafat C, Sztrymf B, Dreyfuss D, Ricard JD. Use of high-flow nasal cannula oxygen therapy in subjects with ARDS: a 1-year observational study. Respir Care. 2015;60(2):162-9.

8. Nagata K, Morimoto T, Fujimoto D, Otoshi T, Nakagawa A, Otsuka K, Seo R, Atsumi T, Tomii K. Efficacy of high-flow nasal cannula therapy in acute hypoxemic respiratory failure: decreased use of mechanical ventilation. Respir Care. 2015;60(10):1390-6.

9. Frat JP, Thille AW, Mercat A, Girault C, Ragot S, Perbet S, Prat G, Boulain T, Morawiec E, Cottereau A, et al. High-flow oxygen through nasal cannula in acute hypoxemic respiratory failure. N Engl J Med. 2015;372(23):2185-96.

10. Maggiore SM, Idone FA, Vaschetto R, Festa R, Cataldo A, Antonicelli F, Montini L, De Gaetano A, Navalesi P, Antonelli M. Nasal high-flow versus Venturi mask oxygen therapy after extubation. Effects on oxygenation, comfort, and clinical outcome. Am J Respir Crit Care Med. 2014;190(3):282-8.

11. Hernandez G, Vaquero C, Colinas L, Cuena R, Gonzalez P, Canabal A, Sanchez S, Rodriguez ML, Villasclaras A, Fernandez R. Effect of postextubation high-flow nasal cannula vs noninvasive ventilation on reintubation and postextubation respiratory failure in high-risk patients: a randomized clinical trial. JAMA. 2016;316(15):1565-74.

12. Hernandez G, Vaquero C, Gonzalez P, Subira C, Frutos-Vivar F, Rialp G, Laborda C, Colinas L, Cuena R, Fernandez R. Effect of postextubation highflow nasal cannula vs conventional oxygen therapy on reintubation in lowrisk patients: a randomized clinical trial. JAMA. 2016;315(13):1354-61.

13. Mauri T, Turrini C, Eronia N, Grasselli G, Volta CA, Bellani G, Pesenti A. Physiologic effects of high-flow nasal cannula in acute hypoxemic respiratory failure. Am J Respir Crit Care Med. 2017;195(9):1207-15.

14. Fraser JF, Spooner AJ, Dunster KR, Anstey CM, Corley A. Nasal high flow oxygen therapy in patients with COPD reduces respiratory rate and tissue carbon dioxide while increasing tidal and end-expiratory lung volumes: a randomised crossover trial. Thorax. 2016;71(8):759-61.

15. Pisani L, Fasano L, Corcione N, Comellini V, Musti MA, Brandao M, Bottone $D$, Calderini E, Navalesi P, Nava S. Change in pulmonary mechanics and the effect on breathing pattern of high flow oxygen therapy in stable hypercapnic COPD. Thorax. 2017;72(4):373-5.

16. Pisani L, Vega ML. Use of nasal high flow in stable COPD: rationale and physiology. COPD. 2017;14(3):346-50.

17. Sinderby C, Navalesi P, Beck J, Skrobik Y, Comtois N, Friberg S, Gottfried SB, Lindstrom L. Neural control of mechanical ventilation in respiratory failure. Nat Med. 1999;5(12):1433-6.
18. Doorduin J, van Hees HW, van der Hoeven JG, Heunks LM. Monitoring of the respiratory muscles in the critically ill. Am J Respir Crit Care Med. 2013;187(1):20-7.

19. Beck J, Sinderby C, Lindstrom L, Grassino A. Effects of lung volume on diaphragm EMG signal strength during voluntary contractions. J Appl Physiol (1985). 1998;85(3):1123-34.

20. Beck J, Sinderby C, Lindstrom L, Grassino A. Influence of bipolar esophageal electrode positioning on measurements of human crural diaphragm electromyogram. J Appl Physiol (1985). 1996;81(3):1434-49.

21. Bellani G, Mauri T, Coppadoro A, Grasselli G, Patroniti N, Spadaro S, Sala V, Foti $G$, Pesenti A. Estimation of patient's inspiratory effort from the electrical activity of the diaphragm. Crit Care Med. 2013;41(6):1483-91.

22. Sessler CN, Gosnell MS, Grap MJ, Brophy GM, O'Neal PV, Keane KA, Tesoro EP, Elswick RK. The Richmond agitation-sedation scale: validity and reliability in adult intensive care unit patients. Am J Respir Crit Care Med. 2002; 166(10):1338-44.

23. Tobin MJ. Respiratory monitoring in the intensive care unit. Am Rev Respir Dis. 1988;138(6):1625-42.

24. Barwing J, Ambold M, Linden N, Quintel M, Moerer O. Evaluation of the catheter positioning for neurally adjusted ventilatory assist. Intensive Care Med. 2009;35(10):1809-14.

25. Di Mussi R, Spadaro S, Mirabella L, Volta CA, Serio G, Staffieri F, Dambrosio M, Cinnella G, Bruno F, Grasso S. Impact of prolonged assisted ventilation on diaphragmatic efficiency: NAVA versus PSV. Crit Care. 2016;20(1):1

26. Liu L, Liu H, Yang Y, Huang Y, Liu S, Beck J, Slutsky AS, Sinderby C, Qiu H. Neuroventilatory efficiency and extubation readiness in critically ill patients. Crit Care. 2012;16(4):R143.

27. Schmidt M, Kindler F, Cecchini J, Poitou T, Morawiec E, Persichini R, Similowski T, Demoule A. Neurally adjusted ventilatory assist and proportional assist ventilation both improve patient-ventilator interaction. Crit Care. 2015;19:56.

28. Hedenstierna G. Esophageal pressure: benefit and limitations. Minerva Anestesiol. 2012;78(8):959-66.

29. Baydur A, Behrakis PK, Zin WA, Jaeger M, Milic-Emili J. A simple method for assessing the validity of the esophageal balloon technique. Am Rev Respir Dis. 1982;126(5):788-91.

30. Mauri T, Alban L, Turrini C, Cambiaghi B, Carlesso E, Taccone P, Bottino N, Lissoni A, Spadaro S, Volta CA, et al. Optimum support by high-flow nasal cannula in acute hypoxemic respiratory failure: effects of increasing flow rates. Intensive Care Med. 2017:43(10):1453-63.

31. Beck J, Gottfried SB, Navalesi P, Skrobik Y, Comtois N, Rossini M, Sinderby C. Electrical activity of the diaphragm during pressure support ventilation in acute respiratory failure. Am J Respir Crit Care Med. 2001;164(3):419-24.

32. Telias I, Brochard L, Goligher EC. Is my patient's respiratory drive (too) high? Intensive Care Med. 2018;

33. Dres M, Schmidt M, Ferre A, Mayaux J, Similowski T, Demoule A. Diaphragm electromyographic activity as a predictor of weaning failure. Intensive Care Med. 2012;38(12):2017-25.

34. Barwing J, Pedroni C, Olgemoller U, Quintel M, Moerer O. Electrical activity of the diaphragm (EAdi) as a monitoring parameter in difficult weaning from respirator: a pilot study. Crit Care. 2013;17(4):R182.

35. Thille AW, Harrois A, Schortgen F, Brun-Buisson C, Brochard L. Outcomes of extubation failure in medical intensive care unit patients. Crit Care Med. 2011;39(12):2612-8.

36. Sassoon CS, Light RW, Lodia R, Sieck GC, Mahutte CK. Pressure-time product during continuous positive airway pressure, pressure support ventilation, and T-piece during weaning from mechanical ventilation. Am Rev Respir Dis. 1991;143(3):469-75.

37. Mancebo J, Isabey D, Lorino H, Lofaso F, Lemaire F, Brochard L. Comparative effects of pressure support ventilation and intermittent positive pressure breathing (IPPB) in non-intubated healthy subjects. Eur Respir J. 1995;8(11):1901-9.

38. Rochwerg B, Brochard L, Elliott MW, Hess D, Hill NS, Nava S, Navalesi P, Antonelli M, Brozek J, Conti G, et al. Official ERS/ATS clinical practice guidelines: noninvasive ventilation for acute respiratory failure. Eur Respir J. 2017; 50(2):1602426-46.

39. Mauri T, Langer T, Zanella A, Grasselli G, Pesenti A. Extremely high transpulmonary pressure in a spontaneously breathing patient with early severe ARDS on ECMO. Intensive Care Med. 2016;42(12):2101-3.

40. Lourenco RV, Cherniack NS, Malm JR, Fishman AP. Nervous output from the respiratory center during obstructed breathing. J Appl Physiol. 1966; 21(2):527-33. 
41. Yan S, Lichros I, Zakynthinos S, Macklem PT. Effect of diaphragmatic fatigue on control of respiratory muscles and ventilation during $\mathrm{CO} 2$ rebreathing. J Appl Physiol (1985). 1993;75(3):1364-70.

42. Bradley GW, von Euler C, Marttila I, Roos B. Steady state effects of CO2 and temperature on the relationship between lung volume and inspiratory duration (Hering-Breuer threshold curve). Acta Physiol Scand. 1974;92(3):351-63.

43. Bateman NT, Leach RM. ABC of oxygen. Acute oxygen therapy. BMJ. 1998; 317(7161):798-801.

Ready to submit your research? Choose BMC and benefit from:

- fast, convenient online submission

- thorough peer review by experienced researchers in your field

- rapid publication on acceptance

- support for research data, including large and complex data types

- gold Open Access which fosters wider collaboration and increased citations

- maximum visibility for your research: over $100 \mathrm{M}$ website views per year 